\title{
Data shown
}

\author{
'Data not shown' is an outdated caveat that obscures the transparency of a scientific report and weakens the peer \\ review process.
}

$T$ echnology and competition perpetually raise the bar for the quality and quantity of experimental data that authors must include to publish a high-impact manuscript. Almost uniformly, journals have amended their formats to accommodate the increased volume of data while maintaining page restrictions by providing the supplementary information option online for authors and readers. Despite these changes, many authors still rely on the caveat of 'data not shown'. At Nature Chemical Biology, we discourage the use of this phrase and the omission of important data for two major reasons. First, the exclusion of essential data undermines the peer review process, and second, readers need access to data to form independent opinions about and to replicate the results of published papers. Thus, we suggest that the time for 'data not shown' has passed.

To accommodate the natural progression of both standards for research and broadened accessibility to technologies, we encourage authors to replace detailed methodological descriptions with relevant citations whenever possible. Indeed, some methods have become so customary (for example, PCR and flash column chromatography) that they no longer merit a specific citation. Data derived from established methods and specific details about the authors' system (for example, primer sequences), however, must be included. For additional details on presenting and consolidating methods, see our Guide to Authors (http://www.nature.com/nchembio/pdf/nchembio_gta.pdf).

Likewise, technological advances leading to the emergence of large datasets have changed the way some data are reported by removing a subset of primary data from papers altogether and concentrating them in data repositories. Following the postgenomic explosion of high-throughput platforms such as genome sequencing, RNA expression profiling and proteomics, scientists and publishers reached a consensus that these data should be publicly available but should not flood journal pages. Nature journals uniformly require deposition of large-scale datasets into existing community-endorsed databases. It remains important, however, that editors and referees have access to these data. We recognize the right of a referee to request any data that are integral for their evaluation of a manuscript and may contact authors to obtain missing files. For more details on our data sharing and database deposition policies, see http://www.nature.com/authors/ editorial_policies/availability.html.

We urge authors of Nature Chemical Biology manuscripts to include all relevant data at the time of initial submission so that they are available for editorial assessment and peer review, and to prevent delays in the final acceptance of their manuscripts. We request that authors familiarize themselves with our editorial policies, but we highlight several aspects that are particularly relevant to most Nature Chemical Biology authors.
For manuscripts that include macromolecular structures, authors must deposit their coordinates in the Protein Data Bank and complete our standard tables for reporting crystallographic or NMR data (downloadable word templates are available at http://www.nature. com/nchembio/authors/submit/index.html\#ch). We are committed to rigorous characterization of the chemical entities in our published papers. Authors must identify the source for known compounds and provide chemical characterization data for all new compounds utilized in their manuscript, which minimally includes ${ }^{1} \mathrm{H}$ NMR, protondecoupled ${ }^{13} \mathrm{C}$ NMR and high-resolution mass spectrometry data. Authors can view guidelines for compound characterization at http:// www.nature.com/nchembio/authors/submit/index.html\#ch. Finally, interpretation of quantitative data must be supported by accepted statistical analyses. Our website provides advice for selecting the proper statistical test and a checklist to help authors avoid common errors (http://www.nature.com/nchembio/authors/submit/index. html\#statistical).

We encourage authors to eschew other situations where data that are relevant to the manuscript or its interpretation are omitted, as these seemingly benign cases can prolong the peer review process. For example, authors often mention negative data that are not readily translated into a meaningful figure or table as 'data not shown'. Mentioning these kinds of data in the text constitutes an assertion of the data, and therefore 'data not shown' is unnecessary. Situations where authors refer to and depend on data that are neither published nor available in the current manuscript ('manuscript submitted', 'manuscript in preparation' or 'unpublished observations') can be more problematic. Manuscripts submitted elsewhere that are related to those under consideration with us must be included for editorial and referee assessment. In cases where the editors and referees concur that data directly impact the current manuscript, we may request that they be incorporated. 'Personal communications', which also qualify as 'data not shown', are allowed with written confirmation from the source, but we discourage authors from relying on any unavailable data to draw their conclusions.

Given the option for including supplementary information online, the existence of public databases and the fundamental importance of a robust peer review process, we conclude that the use of 'data not shown' and other caveats that exclude relevant data from the hands of editors, referees and readers is no longer appropriate. Eliminating these caveats will streamline the peer review process for authors and referees while preserving the rigor of scientific publications. The benefit to the scientific community at large justifies the extra individual effort required to break this outdated habit. 